\title{
TENSEGRIDAD COMO CRÍTICA AL ABUSO DE LAS FORMAS ESTABLES
}

RAFAEL BENEYTEZ VÍCTOR MANUEL CANO
PROFESOR, ETSAM, UNIVERSIDAD POLITÉCNICA DE MADRID, ESPAÑA. DOCENTE, ETSAM, UNIVERSIDAD POLITÉCNICA DE MADRID, ESPAÑA.
FIG 1

Toguna o Casa de la Palabra.

País Dogón, Malí.

Fotografía de Luciano Napolitano.

\section{FIG 2}

Cartografía de la huida de Rudolf Vbra y Alfred Wetzler en Auschwitz-Birkenau: situaciones espaciales a partir del entendimiento y la visibilización de las estructuras de relación y tensión de los cuerpos en el espacio.

Dibujo de Víctor Manuel Cano, 2013.

\section{FIG 3}

Cartografía de la revuelta de los sonderkommandos en Auschwitz-Birkenau. Dibujo de Víctor Manuel Cano, 2013.
El mundo de reglas, recetas y costumbres $\mathbf{1}$ son respetadas en las sociedades más ancestrales. No han podido ser tomadas a la ligera ni entre los sabios de las comunidades más arcaicas.

\section{AIRE Y TENSIÓN O LA DEMOCRACIA DE LAS NUBES}

E n las sociedades del pueblo Dogón, en Malí, existe la casa de la palabra o Toguna. La Toguna es el lugar donde se reúnen a diario los ancianos y sabios del pueblo, para discutir los asuntos y disputas, contar cuentos y establecer reglas. La arquitectura que representa este acuerdo es una estructura de pilares, cubierta con una techumbre plana, sobre la que se depositan ocho gruesas capas de paja de mijo, en representación de los ocho ancestros del pueblo Dogón (fig. 1). La Toguna es el lugar de las reglas y las normas, pues no hay en el mundo Dogón otra cosa que la tradición oral para transmitir las leyes que rigen el equilibrio social. Son, estas recetas, la peculiaridad de mundo de lo humano. $Y$ a ellas se somete cada miembro, pues son las formas y las leyes quienes permanecen, mientras los mortales van y vienen. Esta estructura de reglas que definen los sabios es la que conduce las pautas para la acción social y es a lo que también se designa como "nomotopo". Un neologismo utilizado por diversos autores del pensa-

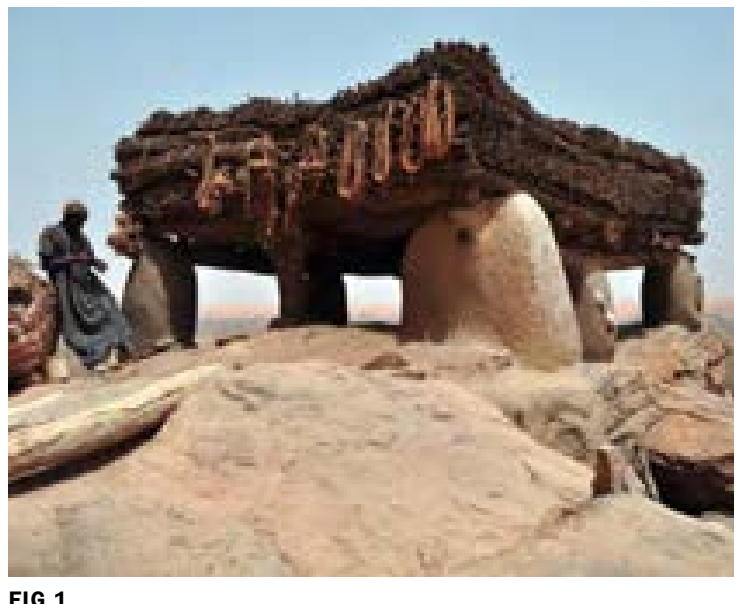

FIG 1

miento contemporáneo y que tiene especial interés, para lo que este artículo pretende, a través de las ideas de Peter Sloterdijk.

Para Peter Sloterdijk, filósofo y catedrático de la Escuela de Arte y Diseño de Karlsruhe, toda la construcción de la modernidad se asemeja metafóricamente a una construcción de esferas. Así presenta al mundo moderno en su trilogía Esferas, una obra monumental escrita en tres tomos donde la esfera como estructura geométrica, formal y superficial domina todas las ideas presentadas. Para ello, acuñadas en una serie de neologismos acabados en la palabra

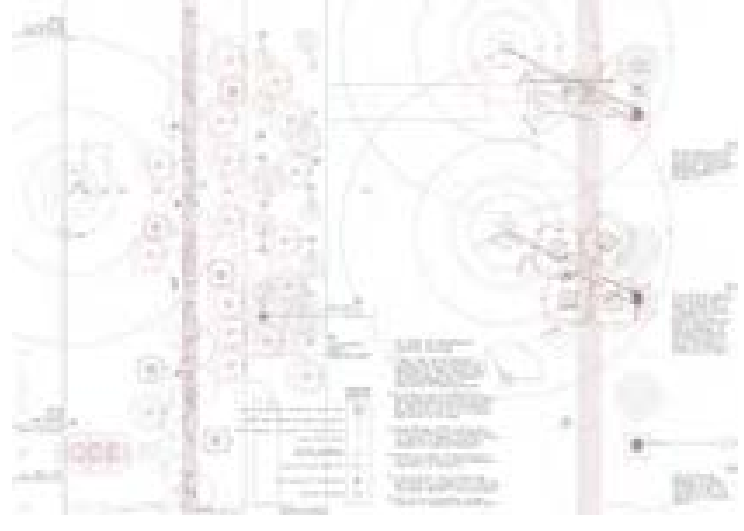

FIG 2

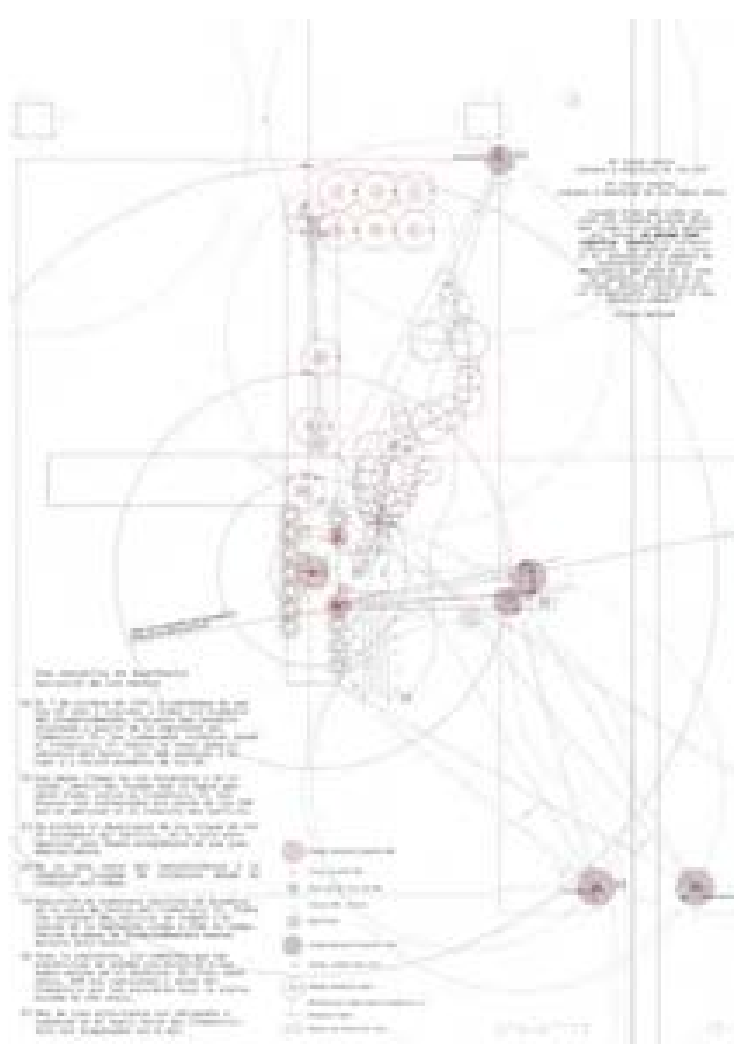

FIG 3

esfera, las ideas planteadas reflexionan sobre la organización temática de los modos del saber moderno. En Esferas III, el último volumen, presenta el mundo como idea de lo puramente humano bajo el neologismo de antropo-sfera que se refiere al hombre inserto en una construcción a la que llamamos mundo.

De acuerdo a Sloterdijk este ser humano se relaciona con el mundo a través de nueve dimensiones a las que designa topoi y que describen cómo el hombre interactúa con él desde su fisicidad hasta su psicología, desde su individualidad hasta su colectividad, desde su relación con el medioambiente hasta sus 
La relación entre una lógica estructural que plantea la existencia de equilibrios e interdependencias y sistemas de organización social abiertos y no jerárquicos remite a las afirmaciones de Sloterdijk respecto a los vínculos entre los paradigmas de la estática y los del Estado.

PALABRAS CLAVE: ARQUITECTURA - TEORÍA Y CRÍTICA, NOMOTOPO, METAESTABILIDAD, TENSEGRIDAD, PRESOSTÁTICA.
Sloterdijk discussed the links between paradigms of static and State; that would be a key reference to understand connections between a structural logic that depends on the existence of balanced interdependencies and open and non-hierarchical social schemes.

KEYWORDS: ARCHITECTURE - THEORY, NOMOTOP, METASTABILITY, TENSEGRITY, PRESOSTATICS. English text in p. 30
FIG 4

Bandada de estorninos. Roma. Italia. 2008

Fuente: archivo de los autores.

\section{FIG 5}

Fotograma de Mirror, Andrei Tarkovsky, 1974.

Fuente: http://revistacruce.com/artes/ hacia-un-verdadero-cine-poetico.html
El quirotopo o el mundo a mano, el $\mathbf{2}$ fonotopo o aquél generado por la campan vocal, el uterotopo o el ámbito maternal, el termotopo o las experiencias del mundo del confort, el erototopo o lugar de transferencias de las energías eróticas, el ergotopo o fuerza paternal o sacerdotal, el alethotopo o grupo del aprendizaje en el entorno de la verdad, el thanatotopo o mundo de los muertos y el nomotopo, que representa la relación de acuerdos que tiene como contexto "la política".

A partir de la expresión Tensión Integrity 3 Structures de Buckminster Fuller. construcciones políticas. A través de ellos recorre de manera sistemática todos los rincones de la historia moderna, recuerda historias antiguas y nos proyecta hacia un futuro alumbrado por un pasado reciente cargado de acontecimientos fascinantes. De los nueve topo $^{2}$, el que nos interesa profundizar es el nomotopo "que vincula recíprocamente a los coexistentes por 'costumbres' comunes, por reparto del trabajo y expectativas recíprocas, con lo que, por el intercambio y el mantenimiento de la cooperación, aparece una tensegridad imaginaria, una arquitectura social compuesta de expectativas, apremios y resistencias mutuos, en una palabra: una primera constitución" (Sloterdijk, 2004, p. 280).

En esta breve definición la democracia es una de las formas de construcción política en la que la idea de "tensegridad" cobra especial valor, pues su mayor interés radica en mantener las continuidades entre todos los individuos sin importar su condición y clase social (figs. 2 y 3). Fijémonos en que tal continuidad se produce a través de un campo de relaciones entre individualidades que participan en un conjunto unifi-

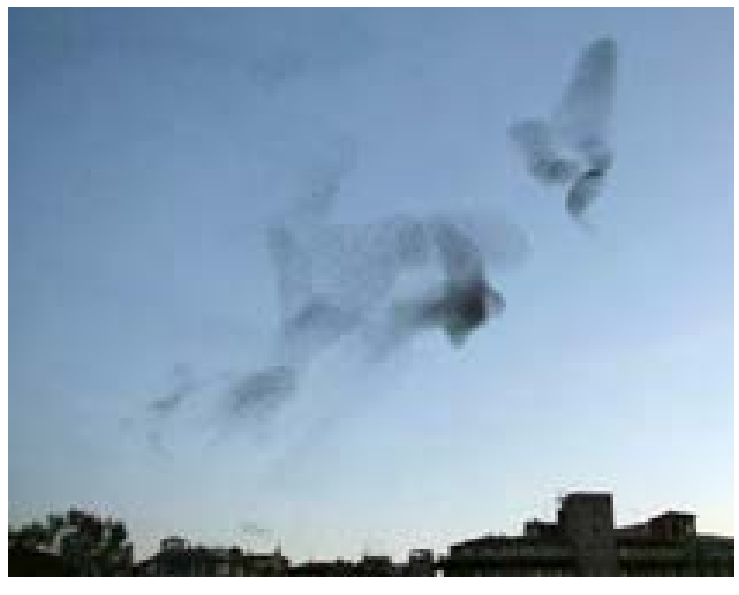

FIG 4

cado y unificador. Bandadas de pájaros, bancos de peces y las nubes, en tanto aparatos meteorológicos, son expresiones formales cuyo argumento principal se inscribe en un ámbito de relaciones de reciprocidad en el interior de un sistema que, sin ser exactamente estructuras tensegríticas, anticipan un valor mayor por sobre el valor de su apariencia formal. A partir de esto, las tensegridades son estructuras de relaciones cuyas expresiones formales se hacen legibles analizando el comportamiento de los elementos que las organizan como sistema. Sistemas compuestos tanto por suma de partículas aisladas, como por la suma de partículas pertenecientes a un conjunto con el que se relacionan activamente (fig. 4 ).

Las sociedades son las primeras estructuras, sistemas o conjuntos que necesitan un claro sentido del orden. Tienden a la organización continua de un sistema estabilizado bajo el cual, el colectivo acompañado de una atmósfera de tensión, consigue actuar solidariamente en un rol de cargas y beneficios que mantienen el equilibrio. Sin embargo, las lógicas de forma que las representan tanto en el campo de la ciencia como explícitamente en la arquitectura, tardaron en estar a la altura de tal construcción sistémica. Sólo pudieron aparecer en el momento en que la técnica y las ideas se unieron en el ámbito de las estructuras presostáticas y en el de las estructuras tensegríticas, aquellas en las que una fuerza continua y modulable mantiene, al menos, la tensión mínima del conjunto que hace prevalecer el equilibrio. Cuando esta fuerza se disipe, toda la organización estructural se desvanecerá en el aire. En el caso de las estructuras presostáticas (fig. 5) es el aire quien hace la doble función de medio de vida y ámbito estructural. En cierto modo

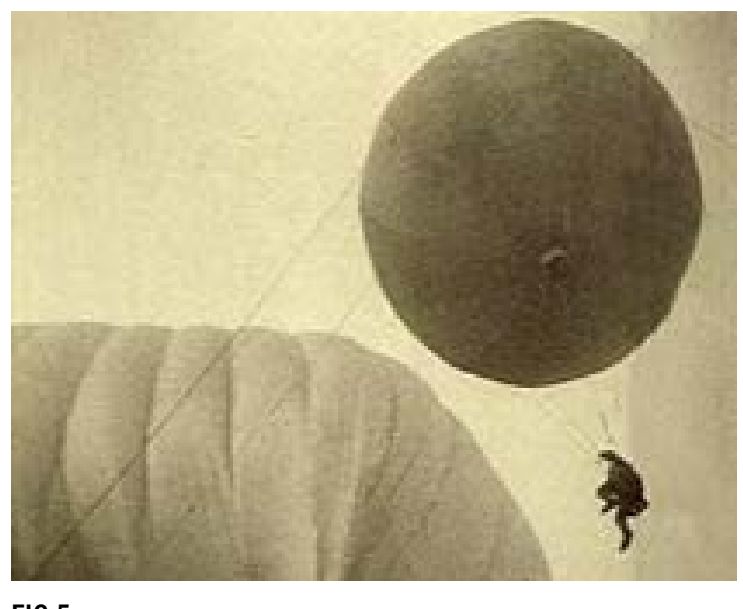

FIG 5

representa lo que podríamos llamar una estructura respirable, o quizá, un medio de vida con posibilidad de configurar una estructura autosustentante. El caso de la tensegridad ${ }^{3}$ es un principio regulador de cargas de tracción y compresión que almacena energía en el interior de un entramado de vectores y fuerzas que estabilizan el conjunto formal. Gracias a la existencia de una tensión interna se produce un estado de equilibrio. Esta forma estructural se resuelve sobre un entramado de barras y cables cuya organización espacial permite mantener, bajo determinadas reglas de orden, un equilibrio global 
Seiltänzer (Equilibrista). Litografía, 43,2 x 26,8 cm, Paul Klee, 1923.

Fuente: Zentrum Paul Klee, Berna.

Fuera de las discusiones de autoría de $\mathbf{4}$ la primera estructura tensegrítica, cabe señalar que para el caso de Ioganson se realizan dos cosas de manera simultánea y sin antecedentes: un principio de estática revolucionario y un estado político social revolucionario.

Una alternativa que sin superar el problema 5 de la gravedad como tema hegemónico en arquitectura, planteaba un desplazamiento de los centros de gravedad y de las estructuras formales buscando un desequilibrio visual o un dinamismo de la forma. En definitiva, una primera crítica a la estática.

Las vanguardias rusas del arte estuvieron $\mathbf{6}$ implicadas en esta transformación técnica y productiva llegando a expresar su momento

álgido entre 1923 y 1926 en la Exposición Internacional de Artes Decorativas e Industrias Modernas de París de 1925 conquistando varias medallas.

Que el estrés colectivo sea la pauta común 7 para el funcionamiento estable, la reacción a lo inesperado o la adaptación a lo móvil, permite pensar en cómo o bajo qué condiciones se realizan los aumentos constantes de tensión interna para ser sobrellevados sin la fatigosa vivencia de la tensión continua. Las instituciones y las rutinas son modos de operar social para la sublimidad de estos estados continuos de tensión. Se asume que con estos dos modos de regular las tensiones continuas se sacrifica la

libertad de elección y lo que desaparece es la sobrecarga del exceso de libre elección del individuo que pretende el estado de equilibrio. Así, este individuo huye tanto de la exigente tarea de la originalidad de sus actos como de la miseria de la falta de formas. Descargados de la sobrepresión continua, liberan energía para poder afanarse con otras tareas. A los arquitectos, toda vez que emprenden una idea formal, les toca argumentar hasta el punto de la realización cada paso en una urdimbre de relaciones productivas enfocadas hacia el mismo objeto. La división del trabajo planteada por la modernidad, nos obliga a la estructuración de tensiones internas ordenadas en un patrón autoestable en la que todos los integrantes saben de sobra la dependencia de unas y otras para el logro colectivo. La complementariedad entre las partes significa demanda de tensión que unifica la división del trabajo entre agentes coligados. Que esta modulación de cargas de aumento, disminución y tensión haya sido eficaz, se hace indispensable frente

a todo lo realizado con éxito, dado que tales realizaciones son fruto de un hacer tensionado entre sensibilidades de diversas disciplinas y aunadas hacia una belleza buscada a través de los rigores disciplinarios de muchas individualidades coligadas bajo la misma tensión en cada paso.

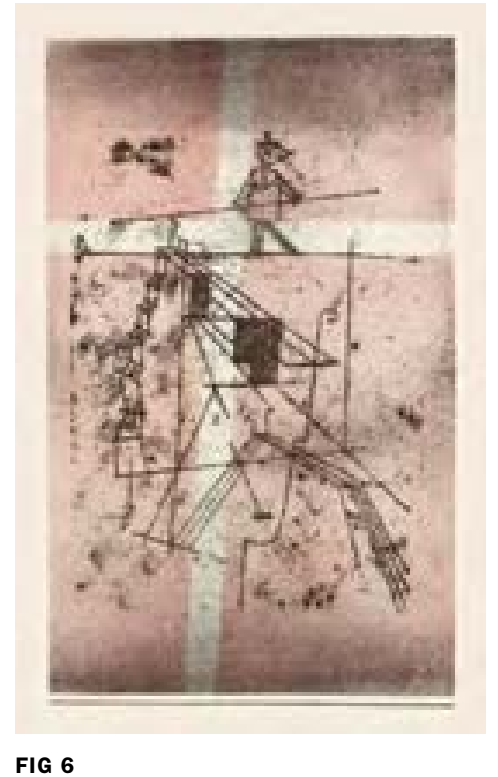

sin excepciones de modo que todo elemento trabaja solidariamente con la totalidad del sistema. La similitud de estos principios con el orden de las estructuras sociales -incluso aquellas más ancestrales, como las del pueblo Dogón- radica en parte importante en la mantención de la estabilidad por adaptación a lo móvil, tal como Sloterdijk lo presenta.

Tales formas paradigmáticas, que aparecerían a principios de los años 1920, hicieron simultáneamente visible la relación entre el estado y la estática en el caso de Karl Ioganson. También lo harían a través de Kenneth Snelson y Buckminster Fuller aproximadamente en la misma época. Sin embargo, en el caso de Ioganson lo que resulta valioso es el estrechamiento de la relación entre la situación política del estado y los nuevos conceptos de estática ${ }^{4}$. Hasta entonces, el repertorio formal de la estática estaba limitado a estructuras de fuerte componente gravitatoria y baja energía potencial.

En 1920 la búsqueda colectiva del servicio de la técnica y el arte al espíritu revolucionario ofreció en el contexto de los vkhutemas soviéticos -Talleres Superiores Artísticos y Técnicos del Estado- una alternativa a la estática clásica: estructuras espaciales que, fortaleciendo el discurso constructivista, hacían con la ligereza y el colectivo la imagen de contrapunto al peso material que representaba a la aristocracia zarista ${ }^{5}$. Tras varias exposiciones y trabajos intensos sobre este problema, Karl Ioganson proponía una serie de esculturas, llamadas construcciones espaciales, entre las que se incluía una estructura tensegrítica. La Rusia postrevolucionaria fue impulsada en el camino técnico de la construcción arquitectónica e ingenieril como símil de las deseadas nuevas estructuras sociales de fuerte sentimiento colectivo y colaborativo que huían de un mundo de reglas severas. Dichas reglas acabaron en la hambruna del pueblo y en el comienzo de la Revolución Rusa en febrero de 1917. La fuerte voluntad por reconstruir un sistema de reglas y leyes hizo especial hincapié en el arte de la producción. Lenin, implicado en la reconstrucción económica, dictaba pautas para reconfigurar las fuerzas nacionales de producción sobre un claro impulso de cambio. El reflejo de tales pautas transformó las instituciones docentes bajo un laborioso proceso de revisión crítica del sentido del arte y la técnica, comenzando por las Escuelas de Artes y Oficios para acabar con los Estudios Básicos y los vkhutemas. Estos funcionaron durante casi una década como una estructura docente autorregulada y apoyada en una actividad evolutiva e intensamente inquieta ${ }^{6}$. En este contexto, cabe insistir que el valor que adquiere el paradigma de la Revolución Rusa está precisamente en que tanto estado como estática tuvieron un reflejo mutuo de intensa interacción que acabó ofreciendo el paradigma de la tensegridad.

Sloterdijk insiste en esta relación entre los paradigmas de la estática y del Estado, o dicho de otro modo, en la relación íntima entre la tensegridad y la organización político y social, y subraya las nociones mencionadas por Pierre Legendre, filósofo del derecho y psicoanalista nacido en Francia en 1930. Legendre habla "del derecho y del Estado como magnitudes que sólo pueden sostenerse por medio de un andamio moral o construcción normativa (échafaudage, montage)". El origen etimológico común de las palabras estado y estática permite hablar de que ambos son artes de la construcción: la construcción de normas y la construcción de edificios. Cuando se establecen las pautas de orden social, la empatía que mantienen con las formas tensegríticas o presostáticas viene de comprender la existencia de fuerzas recíprocas; fuerzas organizadas como conjunto de normas y leyes que son necesarias para constituir el equilibrio. Se puede entender que en ellas, una presión repetitiva coloca a cada miembro en el mismo umbral de estrés de cargas que, a nuestro modo de ver, se expresa en la reciprocidad de cargas y derechos, así como en corresponsabilidades para el equilibrio del sistema político, ético y moral. Esta llamada de atención hacia la relación entre carga o estrés colectivo con la idea de cultura, no permite la libertad del individuo colaborador. Más bien esto es un sometimiento a las leyes grupales sin excusa alguna de laxitud dentro de unos ratios mínimos y máximos tolerables. Sin esta condición no existirá ni estática ni estado. En el caso de la estructuras tensegríticas como paradigmas de la estática, no se mantendrán erguidas y para el caso de las estructuras político-sociales, no se mostrarán apaciguadas. ${ }^{7}$

Por todo lo anteriormente expuesto, la conclusión indica que pertenecer a una unidad de tensegridad es estar al día de las tensiones recibidas, sensibilizado coordinadamente y de manera cooperativa con el conjunto sistémico y, además es ser capaz de reaccionar ante la tensión del conjunto. Una deformación del equilibrio global y/o del orden colectivo implica que hay quien se relaja y quien se sobre esfuerza. Un estado de sobresfuerzo por parte de algunos miembros lleva al conjunto a una rigidez creciente que pone en riesgo la integridad del conjunto por rotura de los elementos sobrecargados. Para el caso de la estática, cuando este aumento de rigidez se produce, también se estará observando la expresión formal de tal sobresfuerzo a través de la apariencia formal del conjunto erigido. 


\section{CONFORMACIÓN, METAESTABILIDAD Y CUERPO SIN ÓRGANOS}

El artista suizo Paul Klee tenía una sensibilidad fuera de lo común hacia una naturaleza que, desde su percepción, no dejaba de moverse. Klee afirmaba que la naturaleza era un gran móvil. Este, en constante movimiento, era ejemplificado en sus pinturas y en sus títeres a través del balanceo de las olas, el crecimiento de las plantas, la génesis de un sonido o mediante las tensiones que el alambrista sufre en su paseo por las nubes (fig. 6). En 1924 a través de su Teoría elemental de lo creativo introduce el concepto de conformación ${ }^{8}$ que tiene vital importancia para el entendimiento de su obra ya que sustenta sus ideas sobre la inestabilidad de la forma y sobre el perpetuo movimiento de las cosas. La conformación podría explicarse como el constante proceso de evolución de la forma. Esto quiere decir que la forma no es estática, sino que se produce teniendo en cuenta el tiempo y el devenir. Durante su época de maestro de la Bauhaus hacía hincapié en que dibujar era transmitir el devenir de la forma (Klee, 2013). La forma deja de ser una mera silueta o contorno estable para ser un proceso de transformación en el tiempo y sensible a todas las escalas.

Para finalizar, Klee lo explicará de una manera muy directa:

La conformación determina la forma y por tanto está por encima de ella. Por eso la forma nunca ni en ningún lugar (...) debe ser considerada un resultado, un final, sino génesis, devenir. Buena es la conformación. Mala es la forma, la forma es final, es muerte. La conformación es movimiento, es acto. La conformación es vida. (Klee, 2013, p. 56).

En su primera propuesta hablará sólo de 9 la individuación física existiendo también la vital y la psíquica como el caso de resolución de un sistema metaestable. Tal propuesta la formula ilustrando estados de un sistema como la sobrefusión o la sobresaturación que presiden la génesis de los cristales. Considerándolos claros para dar solvencia al concepto de metaestabilidad, no son lo suficientemente complejos para abarcar el problema de la individuación de manera exhaustiva. La metaestabilidad es un tipo de estabilidad débil que progresa hacia fuertes estados de estabilidad cada vez que se produce una evolución significativa del ser que la conlleva. Esta implica un estado evolutivo y continuo, esto es, la presencia del devenir.

Ahí radica nuestra crítica a la estabilidad, 10 en la imposibilidad de ontogénesis, es decir, en la imposibilidad de que el ser se construya mediante un proceso. En la individuación física la materia pasa a ser esa especie de proceso activo de devenir constante, originándose un objeto de haecceidad, aquel objeto que tiene propiedades relacionales y no sustanciales.

Gilbert Simondon en su libro La indivi- $\mathbf{1 1}$ duación a la luz de las nociones de formay de información explicará dentro del primer apartado del primer capítulo la indivi-

duación física aplicada a la formación del ladrillo, rechazando la dualidad hilemórfica incorporando las nociones de energía y devenir para el entendimiento de este proceso continuo (Simondon, 2009, p. 48-56).
Por otra parte, Gilbert Simondon, filósofo francés, abordó el problema de la estabilidad desde el campo de la física, la química y la biología, y materializó con ello su tesis doctoral La individuación a la luz de las nociones de forma y de información publicada en 1955. En dicho texto se trata el tema de la individuación, un viejo problema de la filosofía y de la técnica que podría definirse como aquello que hace de un individuo algo absolutamente único (Simondon, 2009). De este modo, Simondon se propone un reto faraónico: el intentar explicar cómo devienen las cosas. Para ello comienza haciendo una crítica al esquema hilemórfico de Aristóteles: Simondon ve limitaciones en él. No creía en la propuesta del choque accidental entre materia y forma. Para él no existían accidentes, creía fervientemente en la técnica y en el orden de los procesos energéticos. Por ello, a esta dualidad aristotélica, donde sólo existirían materia y forma durante el proceso de individuación, introduce la noción de energía. Así, podríamos decir que la individuación está formada por la relación entre materia, forma y energía.

En este punto podemos presentar el concepto de "metaestabilidad". Simondon explica que "(la individuación) no ha podido ser pensada y descrita adecuadamente debido a que sólo conocíamos una única forma de equilibrio, el equilibrio estable; no cono- cíamos el equilibrio metaestable; el ser era implícitamente supuesto en estado de equilibrio estable; ahora bien, el equilibrio estable excluye el devenir [...]" (Simondon, 2009, p. 28). Desde esta posición se pueden pensar nuevos sistemas de equilibrio, sistemas que escapan del esquema dualista de movimiento/reposo e inestabilidad/estabilidad, para abrir un campo de posibilidades sobre los estados evolutivos del ser. Esta crítica a la estabilidad clásica introduce una serie de nociones como el de "energía potencial de un sistema, la noción de orden y el aumento de entropía" (Simondon, 2009, p. 28). Se comienzan a valorar los procesos dinámicos en vez de los resultados.

La "metaestabilidad" permite entender que los estados de forma no son ni definitivos ni aislados. Si nos fijamos en las vidrieras de las catedrales góticas, los vidrios son más anchos en su parte más baja. Incluso el cristal, símbolo de forma y equilibrio perfecto, no es eternamente estable, va fluyendo en el tiempo. El vidrio es metaestable y, por lo tanto, va alcanzando diferentes estados de equilibrio a lo largo del tiempo. Quizá lo que más interesante de esta imagen, aplicándolo al problema de representar la tensegridad, es la idea de pensarlo como un sistema metaestable que, cargado de potencialidades, se explica a sí mismo como un conjunto en el que materia, forma y energía preexisten en el sistema y lo van haciendo evolucionar a lo largo del tiempo.

En este sistema hay una concepción global del conjunto que implica una resonancia interna y continua entre todos sus elemento constituidos. La idea de pensarlos separados es más bien fruto de una práctica del método moderno que tiende a separar continuamente los elementos que constituyen los sistemas, pero este principio de individuación reclama un volver a unir y, por ello, un pensar global y continuo. Que las cualidades de uno estén escritas o informadas en las posibilidades del otro y viceversa, necesita considerar la existencia de una energía capacitadora de movimiento o de los cambios de estado para salir de un estado inerte ${ }^{10}$. En la "metaestabilidad" el ser es entendido como un proceso y no como forma estable.

Gilbert Simondon ${ }^{11}$ propone que la idea de "metaestabilidad"se halla en el simple moldeado de un bloque de arcilla. Para él, la formación del ladrillo estará muy lejos de ser representada por aquella dualidad aristotélica entre materia y forma. Simondon propone una bonita metáfora: el esquema hilemórfico sería aquel que percibe el hombre que desde fuera del taller sólo ve el principio y el final del proceso, el paso de arcilla a ladrillo. Y de hecho, para comprender la “metaestabilidad"no bastará con entrar en el taller y trabajar con el artesano, sino que nos haría falta adentrarnos en el molde mismo (Simondon, 2009).

La arcilla es materia a partir de su capacidad de modelarse, de admitir desplazamientos en su composición. Sin embargo, ser modelada no es sufrir desplazamientos arbitrarios, sino ordenar su plasticidad según fuerzas definidas que estabilicen la deformación. Ahora entendemos la crítica de Simondon a Aristóteles, no hay choque accidental, no hay nada arbitrario, todo requiere un riguroso y preciso orden. Preciso orden que, como en la tensegridad, es un 

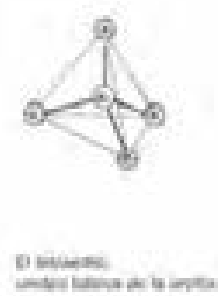

FIG 7

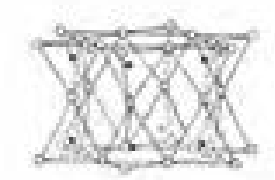

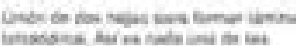

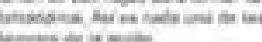

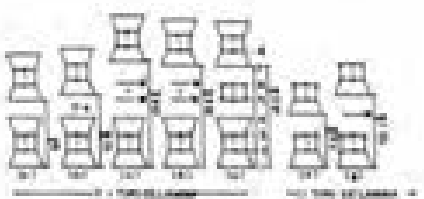

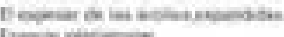

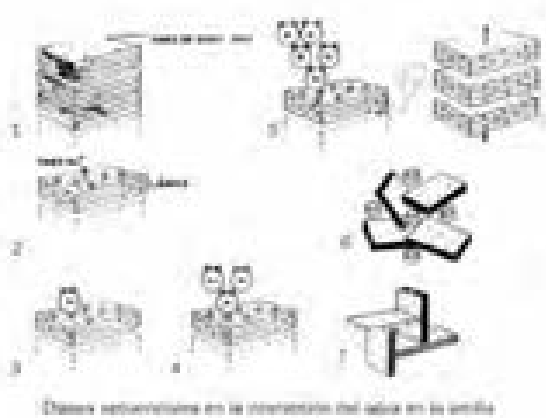

FIG 7

Diferentes estados tensionales y configuraciones de la estructura cristalina de la arcilla.

Fuente: archivo de los autores.
En la esfera del pensamiento de la $\mathbf{1 2}$ química toda estructura que se repite es considerada un cristal. principio básico y fundamental. La estructura compositiva de la arcilla es cristalina ${ }^{12} \mathrm{y}$ está compuesta de "infinitas" láminas de tetraedros que se van relacionando entre sí y que podrían ser analizadas desde el punto de vista de la tensegridad (fig. 7).

El ladrillo es aparentemente estable, pero desde su génesis adquiere la noción de plasticidad. Toda materia es una realidad deformable, es decir, realidad que no es una forma definida, sino todas las formas indefinida y dinámicamente porque en ella se están depositando fuerzas constantemente. Pero no basta con que la arcilla sea plástica, necesita de un obrero que aplique fuerza a la masa, fuerza que la arcilla repartirá a través de la presión de cada lámina en relación con la otra. Todo va cambiando de posición, se va llenando el molde, se va actualizando la energía potencial. El molde no impone la forma, sino que la estabiliza. La relación entre las partículas de arcilla es casi tan fuerte como la relación entre la arcilla y la pared.

Para su total compresión se ha de hacer hincapié en que el molde no impone, pues no es una relación entre materia inerte y forma que viene desde afuera, sino que es un proceso que se basa en la comunicación positiva de algo, como una sociedad de partículas, en interacción recíproca. En un determinado momento aparecerá el límite, es decir, el molde que propicia la imposibilidad de desplazamiento en un sentido determinado. Supongamos que el ladrillo es secado por el calor y queda conformado, la "metaestabilidad" ha encontrado uno de sus posibles estados de equilibrio, pues como ya comentamos, ella es algo así como la existencia de varios estados de equilibrio. Lo que realmente ha sucedido es que las fuerzas se han anulado. La arcilla al final del moldeado es la masa en la cual todas las fuerzas de deformación encuentran, en todas partes, fuerzas iguales y opuestas que la equilibran. Se puede asegurar que gracias a la "metaestabilidad" del sistema, a esa actuación entre energía, materia y forma, se ha podido alcanzar una de las formas finales posibles, donde cualquier acción repercute sobre todos los otros. Es decir, el devenir de cada molécula repercute sobre el devenir de todos los puntos y en todas las direcciones. El moldeado de un bloque de arcilla y la confección un ladrillo, se expresa en este texto como paradigma de un estado tensional complejo y metaestable.

A través del ladrillo hemos visto como Simondon aborda el origen de los objetos físicos, pero también estudiará la individuación de los seres vivos, de lo viviente. Para Simondon, lo viviente implica la noción de interioridad. La interioridad entendida como esas fuerzas que tiene todo ser vivo en su interior. Fuerzas que se hallan en constante movimiento conformando de manera continua, y a cada instante, nuestro cuerpo. Algo como un individuarse constante en el tiempo y en el devenir. El devenir es una transformación, un proceso de cambio de estado que nunca llega a su fin. Las nociones de interioridad y devenir nos desplazan hacia el más excelso alumno de Simondon, el también filósofo francés Gilles Deleuze. Si Simondon explica la "metaestabilidad" desde la física, Deleuze lo hace desde el campo del arte.

Gilles Deleuze dio clases y formó parte de la revolución que tuvo lugar en la universidad parisina de Vincennes. El centro experimental de Vincennes abrió sus puertas en 1968 para reinventar una educación universitaria que sólo era representada por la vieja Sorbona. Nace el 7 de diciembre de 1969 como un refugio intelectual y activo cuya intención era colmar las expectativas de cambio que nunca llegaron a producirse en el Mayo del 68 parisino. Vincennes era como una utopía educativa auto gestionada en base a la reciprocidad de esfuerzos entre alumnos y profesores. Era un sistema tenso donde su existencia se reducía a la solidaridad, convivencia y democracia de todas las partes. No era necesaria una prueba de acceso para la admisión, no existían los exámenes y se promulgaba la autoevaluación. El profesor evitaba las clases magistrales para ser el animador de un trabajo colectivo y los alumnos escogían libremente sus materias. Allí también se discutían las propuestas de los docentes. En definitiva, se trataba de un sistema abierto capaz de adaptarse a las constantes variaciones. En Vincennes se aglutinaron los pensadores más innovadores y radicales de la época; Michael Foucault, Gilles Deleuze, François Lyotard, Jacques Lacan, Michael Serres y Nicos Poulantzas.

Gilles Deleuze era un filósofo enamorado de lo viviente, dejaba de lado los objetos estáticos para centrarse en los cuerpos. Le apasionan los cuerpos porque no dejan de moverse y critica las formas estables desde la noción de cuerpo sin órganos. Cuando Deleuze utiliza este complejo término está haciendo una crítica no tanto a la función que tienen los órganos en el cuerpo, sino a la organización de los mismos, a las formas estables, al orden y previsible funcionamiento que los órganos siguen en las diversas funciones del cuerpo. Digamos que ese orden, ese establecimiento rígido de la manera de ser de un organismo, es ciertamente detestable para el filósofo francés. 
Huevo Dogón. Lugar de intensidades. Fuente: archivo de los autores.

FIG 9

Planimetría Listening Tree, z4z4 AAA arquitectos, noviembre de 2010 Fuente: z4z4 AAA Arquitectos.
Deleuze publica su ensayo sobre el cuerpo sin órganos en el libro Mil mesetas: capitalismo y esquizofrenia. El texto lo encabeza la imagen de un huevo dogón que se explica como espacio de intensidades (fig. 8). El cuerpo sin órganos al que se refiere Deleuze es un huevo. Es un lugar lleno de potencialidades donde todavía no hay forma estable, donde todo son tensiones, fuerzas, energías y multiplicidades. El cuerpo sin órganos ignora la sustancia y la forma basándose en "las relaciones de velocidad y de lentitud entre elementos no formados, y en las composiciones de afectos intensivos correspondientes" (Deleuze, 1988, p. 561).

Desde el campo del arte, Paul Klee también habla de un huevo como lugar de potencialidades: "¿Qué es el huevo? Es evidentemente el cuadro. El cuadro es un huevo, la matriz de las dimensiones" (Deleuze, 2007, p. 40). La matriz es entendida como el lugar donde se engendran las dimensiones, es decir, donde se produce aquello que puede ser mensurable, medido, captado, pero donde aún no hay nada. Como cuerpo metaestable, el huevo es donde se hallan todas esas tensiones internas que conforman el proceso de individuación. Desde esta idea quizá entendamos mejor la máxima de Paul Klee sobre el arte: "No se trata de reproducir lo visible, se trata de volver visible”. ¿Y qué es lo invisible para Klee? Lo invisible son las fuerzas y las energías y para Klee, todo esto es muy obvio; fijémonos en lo que dice en su libro Teoría del arte moderno: “(¿De dónde sacáis tantas fuerzas? Vana pregunta de un bobalicón.) La 'paz en la tierra' es una accidental detención del movimiento de la

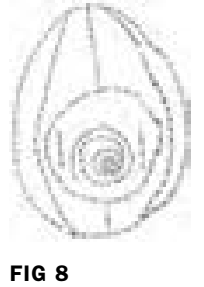

FIG 8

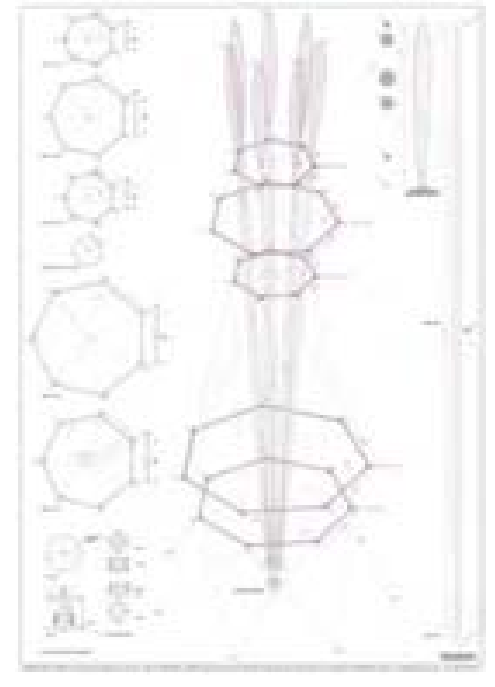

FIG 9 materia. Tener esa fijación por una realidad primera es pura ilusión, [...]" (Klee, 1976, p. 59). Estas fuerzas invisibles Deleuze las encuentra en la obra de Miguel Ángel y sobre ello escribe: "Ahora bien, ¿qué es pintar una ancha espalda de hombre? No es pintar una espalda, es pintar fuerzas que se ejercen sobre una espalda o fuerzas que una espalda ejerce. Es pintar fuerzas, no es pintar formas" (Deleuze, 2007, p. 71). Estas palabras también aluden al pintor irlandés Francis Bacon (1909-1992), cuyo trabajo era otra de las principales debilidades de Deleuze. Bacon pinta fuerzas y cuerpos, cuerpos que son sensibles (hipersensibles) a las fuerzas del entorno. Los cuadros de Bacon no son meras figuras estables, sino cuerpos ${ }^{13}$, entendiendo el cuerpo como el lugar donde se aplican las fuerzas internas y externas, la matriz, el huevo. Cuerpo situado en el devenir y modelado por él.

De nuevo, el relato argumenta cómo la forma estable es puesta en crisis en favor de una capacidad viviente de la forma, alejada de moldes o límites.

No se dibuja (construye) la forma, sino las tensiones (fuerzas y energías) que afectan al cuerpo. Bacon es un apasionado de los cuerpos durmientes; donde parecen no existir fuerzas, como si acaso pudieran verse, Bacon las pinta.

\section{LISTENING TREE 0 EL ELOGIO AL FRACASO}

Las ideas desplegadas a lo largo de los puntos anteriores han ido estableciendo relaciones entre el pensamiento filosófico, la estética, la estática, el estado y la energía, además de elogiar entusiastamente la empatía de sus posibilidades de relación formal. El mismo concepto y sus precisiones descriptivas han ido aclarando las relaciones existentes entre las diversas esferas de conocimiento, e incluso han permitido utilizar unas para explicar a las otras. Finalmente, la tensegridad se ofrece en este artículo como una idea que se expresa eficazmente desde la técnica de erigir estructuras hasta la constitución del Estado. Ambas coinciden mediante la idea de energía potencial que Gilbert Simondon desarrolla para explicar el ámbito de la "metaestabilidad" como un punto intermedio entre lo estable y lo inestable. La idea de "metaestabilidad" ayuda a pensar la tensegridad con mayores herramientas, mejores conceptos y mayor diversidad temática, pues el equilibrio metaestable plantea el equilibrio en un estado transitorio de estabilidades.

Por el contrario, pensar la tensegridad desde el paradigma de la estabilidad y no desde la "metaestabilidad", hace de esta un campo reducido que no incorpora sensibilidad hacia el estado transitorio del que se arma esta composición de cargas, tensiones y actividad interna del sistema. Quizá esta situación no se haga visible, pues al fin y al cabo, cuando se habla en el contexto de la estática y el Estado, se trata de eludir todos los conceptos evolutivos; para la estática, por desearse abusivamente de un estado definitivo y permanente, y para el Estado, por su deseo de preservar la igualdad por encima de la evolución histórica. Pero en ambos casos, y sobre todo para el caso del Estado, la contingencia, la movilidad y las fluctuaciones impiden el equilibrio estable, mientras que para la estática, se convierte en el deseo de realizar la imagen de estabilidad que el Estado no ofrece. Mayoritariamente, la arquitectura resuelve a través de su apariencia tectónica, la paradoja de ofrecer una estabilidad excesiva para un mundo que resulta esencialmente cambiante y realmente frágil.

Ahora, lo deseable es adquirir el compromiso de llevar a cabo un modelo de estructura tensegrítica que ponga en evidencia la necesidad de reciprocidad de fuerzas para la existencia de una estabilidad transitoria, metaestable, operando esta, en el diseño abierto de las relaciones del sistema (fig. 9).

En esta propuesta valdría la pena observar en qué medida el fallo parcial de reciprocidades 
Esquema de nudo para Listening Tree. Fuente: z4z4 AAA Arquitectos.

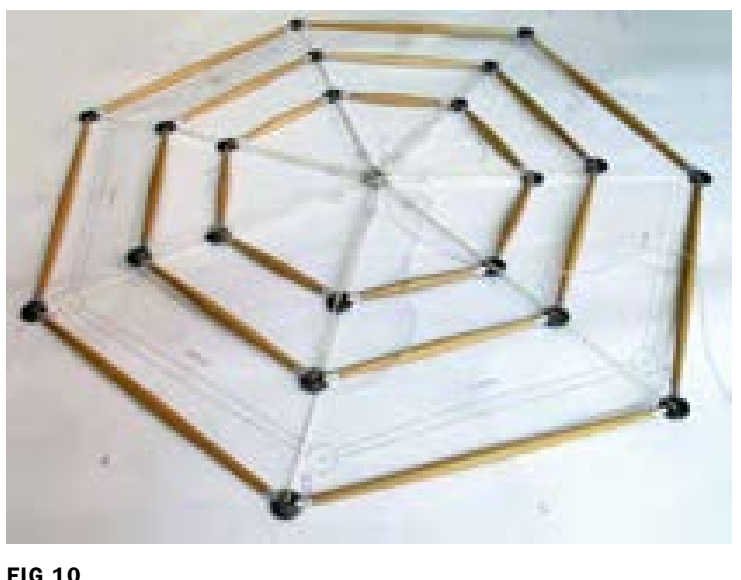

FIG 10

vulneraría el equilibrio total, esto es, el valor de la existencia de un orden y su capacidad de evolución. Si un sistema tensegrítico quisiera tomarse en serio la representación fiel de una estática actualizada a la realidad del mundo, tendría que esmerarse en agudizar su solución constructiva hacia el límite en el que cualquier pérdida de pertenencia al orden sistémico debería provocar el desvanecimiento completo de la unidad tensegrítica. Para ello habría que conseguir un estado de pureza tensegrítica que implicaría trabajar exclusivamente con los siguientes elementos para el esquema de esfuerzos: fuerzas de tracción simple, compresión simple, continuidad de fuerzas de tracción y discontinuidad de fuerzas de compresión. Todo ello establecería un orden no impositivo, sino permisivo de acercamiento de la imagen de la estática a la imagen del estado, ambas frágiles, cambiantes y complejas.

En 1953 Robert Le Ricolais, arquitecto e ingeniero francés, recibía el encargo de diseñar un cañón de largo alcance y alta precisión para el ejército francés. Los cañones de largo alcance se realizan aumentando la longitud del mismo, pero al tiempo lo que aumenta es el peso propio, lo que provoca una desviación de la directriz que pasa de ser recta a ser ligeramente curva, perdiendo precisión. El problema de la precisión se resuelve finalmente buscando un aumento de longitud, consiguiendo al mismo tiempo rigidez y ligereza. Le Ricolais ofreció al problema una solución que consistía en comprimir un mástil mediante un conjunto de cables periféricos. Los cables traccionados unían los dos extremos de la barra y, a lo largo de la directriz, un conjunto de barras perpendiculares al mástil iban ofreciendo al eje una colección de fuerzas que reducían las dimensiones de pandeo, mientras eran comprimidas entre los cables y el propio mástil. Si disminuía sin problemas la longitud de pandeo, la directriz podía ser perfectamente recta y se aligeraba tremendamente el conjunto. Este modelo le ofreció numerosas ideas para realizar puentes, depósitos de agua y torres ligeras entre otros. Entre ellas, y dentro de lo que él desarrolló desde el modelo de los pilares suspendidos, aparecía una torre con un punto de apoyo único comprimido en el suelo y varios traccionados. Esta torre era tremendamente rígida y estaba compuesta por varios mástiles que se abrían hacia arriba siguiendo las directrices de un cono. Mientras estos se abrían hacia arriba, eran rodeados por anillos circulares construyendo un cono en sentido inverso que se conectaban a los mástiles a través de barras radiales y perpendiculares al eje central de la torre. Este no era un modelo tensegrítico, era más bien un sistema comprimido por el peso propio y la fuerza de tracción de una familia de cables periféricos.

En el caso de las esferas tensegríticas, el fracaso de un solo elemento conlleva el fracaso parcial de la forma, ya que la esfera tiene capacidad de respuesta en varias direcciones así como la capacidad inherente a su geometría de responder estructuralmente como un acopio de anillos, una cúpula o un arco. Por estas características, nunca se desvanecería por completo. Por el contrario, un modelo de torre tensegrítica podría ser más apropiado para estimular la empatía formal entre los diversos conceptos enunciados. La idea de torre, aparte de recuperar imágenes atávicas sobre el acto de erigir, simbolizar y elevar, manifiesta a través de su desvanecimiento un derrumbamiento en torno al eje vertical, un descenso de todo el conjunto hacia el punto de apoyo por efecto de la gravedad y, por tanto, una imagen enfática del fracaso, pues el estar erguido es lo que hace de la torre lo que es.

Retomando la propuesta de Le Ricolais anteriormente descrita, sólo habría que transformarla funcionalmente hacia un modelo tensegrítico. Para ello, si eliminamos la conexión entre anillos y mástiles, ocurre que los anillos quedan literalmente suspendidos en el aire y, a su vez, trabajan unitariamente con una rigidez interna proporcional a la rigidez del conjunto. También ocurre que si transformamos los anillos de traza curva en polígonos (fig. 10), la estructura se presenta como conjunto de barras y cables, respondiendo con mayor eficacia a la trayectoria rectilínea de las cargas. A continuación articulamos los nudos, de modo que sólo trabajan a compresión en el plano de los anillos poligonales (fig. 11). Entonces, los cables se organizan en cuatro direcciones opuestas, dos a dos pasando por el centro geométrico de un disco metálico y cuya resultante es igual a la suma de las compresiones de las barras y convergente hacia el centro del conjunto. Si esto se resuelve así, toda la estructura estará predispuesta al fracaso con una aproximación máxima a la estabilidad entre dos extremos opuestos: seguir constituida o yacer descompuesta en el suelo.

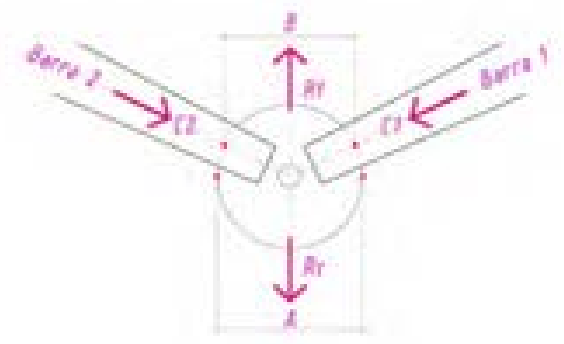

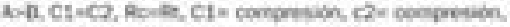

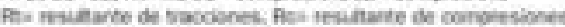


Listening Tree, Cerro de San Pedro, Colmenar Viejo, Madrid. Rafael Beneytez, z4z4 AAA arquitectos, noviembre de 2010. Fotografía de Rafael Beneytez.

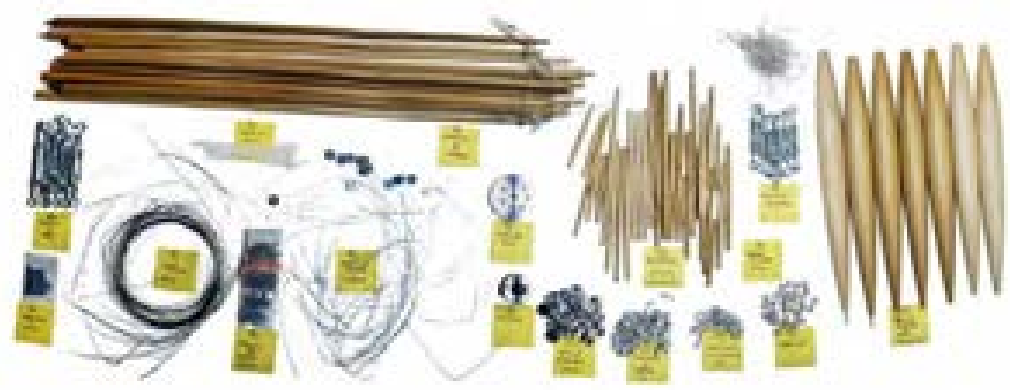

FIG 12

Tras esta remodelación, la propuesta de Le Ricolais, habiéndose banalizado los principios de estabilidad con los que se trabajaba para construir cañones de largo alcance y máxima precisión, habría pasado desde un estado estable a uno "metaestable" y radical. Estado donde se cumpliría exhaustivamente el fracaso ante cualquier deformación milimétrica de la solución inicial, ya que como hemos visto, en los nudos no existe empotramiento alguno y las barras se mantienen comprimidas por un anillo que dimensionalmente no cabe entre los puntos extremos de la barra por una diferencia milimétrica; esta diferencia milimétrica es proporcional a la posible deformación por elongación y retorcimiento de las barras de madera cuando estas cambian de estado higroscópico, como se ilustra a través de Cracking Box de David Nash.

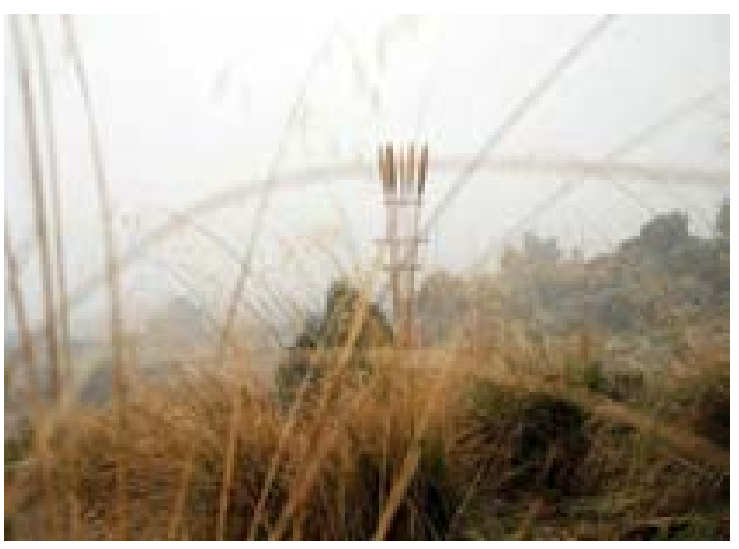

FIG 13

En el año 1992 David Nash permitía que la naturaleza del material acabara sus esculturas de madera. Cracking Box, una caja perfectamente cúbica de madera, era entregada a su evolución formal toda vez que la madera reaccionaba con la humedad ambiente. Al cabo de los meses la caja expresaba el desacuerdo formal con el cubo mediante fisuras, ondulaciones y roturas que hacían de la escultura una doble forma geométrica: la de origen y la de llegada. Esta idea trasladada a la propuesta banalizada de la torre de Le Ricolais, nos lleva finalmente a completar los conceptos, formas y principios para el sistema tensegrítico que anunciábamos como propuesta. Una estructura a la que llamamos Listening Tree (fig. 12).

Listening Tree es una estructura tensegrítica que se construye con madera y metal. La madera utilizada para construir barras se corta con sección de huso, más fina en los extremos que en el centro y con sección longitudinal curva, para responder mejor a la compresión (fig. 11). Una vez montado el conjunto se entrega a su devenir, sabiendo que la madera está cargada de una energía potencial interna y una capacidad activa de transformación en relación a sus estados de humedad relativa. Sus peculiaridades higroscópicas la hacen evolucionar formalmente ante los cambios de humedad ambiente. A su vez, estas barras están coordinadas con la totalidad del conjunto mediante el estrés colectivo que facilitan las tensiones. Estrés, carga o tensión interna que permiten el equilibrio del sistema. Cuando el Listening Tree se introduce en el medioambiente de la Sierra de Guadarrama, al pie del Cerro de San Pedro en Madrid y fuera del taller donde se ha preparado, un proceso de deformación interna de adaptación de la madera al medio, alcanzará lentamente una forma diversa en cada barra (fig. 13). Mientras alcanza el estado de equilibrio, Listening Tree se irá contorsionando en nuevas y diversas formas hasta llegar a límite de su existencia. Aquella en la que se desborda sobre el umbral del último equilibrio posible para saltar como pompa de jabón y desvanecerse en el aire.

\section{CONCLUSIÓN}

El texto planteado en este artículo queda abierto. No plantea de manera cierta la lectura, sino más bien se posiciona en un punto intermedio entre todos los conceptos y temas desplegados para hablar de la misma cosa: la potencialidad de los procesos de formación y, en consecuencia, la instrumentalización del pensamiento para argumentar lo que el título señala como el abuso de las formas estables como práctica asumida.

Para el caso que realiza Listening Tree no sería insignificante entender su fracaso como una forma de expresión de la fragilidad. Fragilidad inherente a la estructura de un sistema que hace visible la pureza como un estado de extraña naturaleza. Una pureza radical sin la cual no puede mantener el equilibrio y que muestra el estado potencial de su estabilidad como una forma transitoria del devenir. Es decir, se ofrece en un equilibrio metaestable entre dos estados posibles: mantener un equilibrio asociado a las condiciones atmosféricas o fracasar entregándose a la gravedad. Así, se muestra como algo hipersensible a los cambios del medio en que habita. Finalmente, Listening Tree se desvaneció en el aire el 16 de noviembre de 2011. ARQ 
RAFAEL BENEYTEZ DURÁN | Arquitecto, Escuela Superior de Arquitectura de Madrid de la Universidad Politécnica de Madrid, 1998 y PhD Internacional Candidate, 2009. Entre 1999 y 2007 colaboró en la oficina de Rafael Moneo como director de proyectos y obra. Fue profesor de Proyectos y director de Estética y Composición en la Escuela Superior de Arquitectura y Tecnología de Madrid y ha sido invitado por universidades en Würzburg, Limerick, Delft, Harvard, Barcelona y Münster. Actualmente es profesor asociado en la Universidad Politécnica de Madrid, desarrolla su tesis doctoral sobre el problema atmosférico en arquitectura y dirige la Oficina Z4Z4 AAA (Architecs Activities Associates).
VÍCTOR MANUEL CANO CIBORRO | Arquitecto, 2012 y Máster en Proyectos Arquitectónicos Avanzados, 2013, Escuela Técnica Superior de Arquitectura de Madrid de la Universidad Politécnica de Madrid. Está vinculado al Grupo de Investigación del Paisaje Cultural y a sus prácticas docentes. Su proyecto fin de carrera ha sido premiado en la V Muestra PFC de la XII Bienal Española de Arquitectura y Urbanismo y su trabajo de fin de Máster obtuvo el Premio Especial en el XII Certamen Arquímedes 2013. La relación entre cuerpo, narraciones espaciales, paisaje y cartografía es el tema de sus investigaciones teórico-prácticas. Es colaborador del estudio de arquitectura Z4Z4 AAA.

\section{REFERENCIAS BIBLIOGRAFICAS}

BERMAN, Marshall. Todo lo sólido se desvanece en el aire. La experiencia de la Modernidad. Buenos Aires, Siglo xxı, 1988 .

BERGSON, Henri. Memoria y vida. Madrid, Alianza Editorial, 1977.

BOURRIAUD, Nicolas. Estética relacional. Buenos Aires, Adriana Hidalgo Editora,

2007.

DELEUZE, Gilles. Francis Bacon: La lógica de la sensación. Madrid, Arena Libros, 2009.

DELEUZE, Gilles. Mil mesetas. Capitalismo y esquizofrenia. Valencia, Pre-textos,

2000.

DELEUZE, Gilles. Pintura: el concepto de diagrama. Buenos Aires, Editorial Cactus, 2007.
DÉOTTE, Jean-Louis. ¿Qué es un aparato estético?: Benjamin, Lyotard, Rancière. Santiago de Chile, Metales Pesados, 2012.

FOUCAULT, Michel. El pensamiento del afuera. Valencia, Pre-Textos, 2008. HEIDEGGER, Martin. El Ser y el Tiempo. Manuel Garrido (Trad.). Madrid, Editorial Tecnos, 2000.

KLEE, Paul. Paul Klee. Maestro de la

Bauhaus. Madrid, Fundación Juan March, 2013.

KLEE, Paul. Teoría del arte moderno. Hugo Acevedo (Trad.). Buenos Aires, Caldén, 1976.

LPPARD, Lucy. Seis Años: La desmaterialización del objeto artístico de 1966 a 1972. Madrid, Akal/Arte Contemporáneo, 2004
MARX, Karl. El manifiesto Comunista. Madrid, Alianza Editorial, 2001. MERLEAU-PONTY, Maurice. El ojo y el espiritu. Madrid, Trotta, 2013. MONOD, Jacques. El azar y la necesidad. Barcelona, Tusquets, 1981 NIETZSCHE, Friedrich. La Gaya Ciencia. Madrid, Edaf, 2002.

RILEY, Terence. Light construction: transparencia y ligereza en la arquitectura de los 90. Barcelona, Gustavo Gili, 1996. ROWE, Colin y SLUTZKY, Robert. Transparency: Literal and Phenomenal. Basilea, Birkhäuser, 1997. SIMMEL, George. Sociología del espacio. Madrid, Alianza Editorial, 1991.
SIMONDON, Gilbert. La individuación a la luz de las nociones de forma y de información. Buenos Aires, Ediciones La Cebra y Editorial Cactus, 2009. SIMONDON, Gilbert. El modo de existencia de los objetos técnicos. Buenos Aires, Prometeo libros, 2008.

SLOTERDIJK, Peter. Esferas I: Burbujas. Microesferología, $3^{\text {a }}$ ed. Madrid, Siruela, 2009 .

SLOTERDIJK, Peter. Esferas II: Globos. Macroesferología, $2^{\text {a }}$ ed. Madrid, Siruela, 2011.

SLOTERDIJK, Peter. Esferas III: Espumas. Esferología Plural. Madrid, Siruela, 2006. VIRILIO, Paul. Estética de la desaparición,

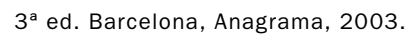

\section{TENSEGRITY AS CRITICAL TO THE ABUSE OF STABLE FORMS}

RAFAEL BENEYTEZ | PROFESSOR, ETSAM, UNIVERSIDAD POLITÉCNICA DE MADRID, SPAIN VÍCTOR MANUEL CANO | INSTRUCTOR, ETSAM, UNIVERSIDAD POLITÉCNICA DE MADRID, SPAIN.

\section{AIR AND TENSION OR}

\section{THE DEMOCRACY OF THE CLOUDS}

In the societies of the Dogon country, in Mali, exists "the house of the word" or Toguna. The Toguna is the place where the village wise men and elders meet daily to discuss business and later tell stories and establish rules. The architecture that represents this program is a structure of pillars with a flat roof covered by eight thick layers of thatch that represent the eight ancestors of the Dogon people (fig. 1). The Toguna is the place of rules and regulations as the oral tradition is the only way to transmit the laws that maintain the social balance in the Dogon world ${ }^{1}$. These recipes are the peculiarity of the human world. Each member is subject to them as they are the rites and laws that remain while the mortal come and go. This structure of rules that the wise men define is the guideline for social action and is also known as "nomotopo." A neologism used by various authors of contemporary thought and has special interest, as claimed by this article, through the ideas of Peter Sloterdijk.

For Peter Sloterdijk, philosopher and professor at the School of Art and Design in Karlsruhe, all of modern construction metaphorically resembles a construction of spheres. This is how he presents the modern world in his Spheres trilogy, a monumental three-volume work, where the sphere as a geometric, formal and superficial structure dominates all the presented ideas. For this, minted in a series of finished neologisms in the word "sphere," the ideas planted reflect on the thematic organization of the modes of modern knowledge. In Spheres III, the last volume, he presents the world as a purely human idea under the neologism anthropsphere that refers to the man inserted into the construction we call world.

According to Sloterdijk this human being relates to the world through nine dimensions that designates topoi and that describe how the man interacts with it from his physicality to his psychology, from his individuality to his collectivity, from his relationship with the environment to his political constructions. Through these, he systematically covers every corner of modern history, remembers ancient histories and projects us toward a future lit by a recent past filled with fascinating events. Of the nine topo $i^{2}$, the one that most interests us most to probe is the nomotop that "reciprocally links the coexisting for common 'customs' through labor divisions and mutual expectations that through exchange and maintaining cooperation an imaginary tensegrity appears, a social architecture composes of social expectations, constraints and mutual resistances, in short: a first constitution" (Sloterdijk, 2004, p. 280).

In this brief definition, democracy is one of the forms of political construction in which the idea of "tensegrity" achieves a special value because its greatest interest lies in maintaining the continuity between all individuals regardless of their status and social class (figs. 2 and 3 ). Notice that such continuity is produced through a field of relationships between individuals that participate in a unified and unifying group. Flocks of birds, schools of fish and banks of clouds, while meteorological instruments, are formal expressions whose main argument is inscribed in a range of reciprocal relationships in the interior of a system that, without being tensegrity structures exactly, anticipate a higher value over the value of its formal appearance. From here, the tensegrities are structures of relationships whose formal expressions are legible by analyzing the behavior of the elements organized as a system. Systems composed by both the sum of isolated particles as by the sum of particles pertaining to a group in which they actively participate (fig. 4).

Societies are the first structures, systems or assemblies that need a clear sense of order. They lean toward the continuous organization of a stabilized system under which the collective, accompanied by an atmosphere of tension, is able to act jointly in a role of checks and balances to maintain balance. However, the logics of form representing them both in the field of science and explicitly in architecture were late in rising to the task of such a systemic construction. They only appeared at the moment in which the art and ideas came together in the pressostatic structures and tensegrity structures, where a continuous and adjustable force maintains the minimum tension of the group that causes balance to prevail. When this force dissipates, all the structural organization will vanish into thin air. In pressostatic structures (fig. 5) it is the air that has the dual function of life agent and structural realm. In some ways, it represents what we could call a breathable structure, or perhaps, way of life with the possibility of creating a self-sustaining structure. The case of tensegrity ${ }^{3}$ is a regulative 
principle of the tensile and compressive energy stored within a lattice of vectors and forces that stabilize the formal assembly. The existence of an internal tension produces a state of equilibrium. This structural form is solved with a lattice of rods and cables whose spatial organization, under certain rules of order, maintains a global balance without exception so that each element works in solidarity with the entire system. The similarity of these principles with the order of the social structures (even the most ancient, like those of the Dogon people) plays an important part in maintaining stability by adapting to the mobile, just as Sloterdijk presents it.

These paradigmatic forms that would appear at the beginning of the 1920's would simultaneously illuminate the relationship between the state and statics in the case of Karl Ioganson. It would also be visible in the work of Kenneth Snelson and Buckminster Fuller around the same time. However, what is most valuable in the case of Ioganson is the narrow relationship between the State political situation and the new concepts in statics ${ }^{4}$. Until then, the formal repertoire of statics was limited to structures with a strong gravitational component and low potential energy.

In 1920 the collective pursuit of the service of art and technique to the revolutionary spirit in the context of the soviet vkhutemas (Professional, Artistic and Technical State Studios) offered an alternative to classical statics: spatial structures that by strengthening the constructivist debate created a countering image of lightness and collectivity to that of the material weight representing the tsarist aristocracys. After working intensely on the problem and several exhibits, Karl Ioganson proposed a series of sculptures, called spatial constructions, which would include the tensegrity structure. Post-revolutionary Russia was driven on the technical path of architecture and engineering construction as a metaphor of the new social structures desired with a strong collective and collaborative feeling that fled a world of severe rules. These rules ended in the starvation of the people and the beginning of the Russian Revolution in February of 1917. The strong will to rebuild a system of rules and laws put special emphasis on the art of production. Lenin, implicated in the economic reconstruction, dictated patterns for reconfiguring the national production forces with a clear impetus for change. The reflection of such patterns transformed the educational institutions with a laborious critical revision process of the meaning of art and technique beginning with the School of Arts and Crafts and ending with elementary education and the vkhutemas. These schools functioned for almost a decade as a self-regulated teaching structure supported by a developing and intensely reckless activity ${ }^{6}$. In this context, it must be noted that the value acquired by the Russian Revolution paradigm is precisely that both the state and statics had a mutual reflection of intense interaction that ended with the offer of the tensegrity paradigm.

Sloterdijk insists in this relationship between the paradigms of statics and the state, or to say another way, in the intimate relationship between tensegrity and social-political organization, and stresses the concepts mentioned by Pierre Legendre, a philosopher of law and psychoanalyst, born in France in 1930. Legendre speaks "of the law and the state as magnitudes that can only be sustained by a moral scaffolding or standardized construction (échafaudage, montage)". The common etymological origin of the words "static" and "state" allows us to speak of both as construction arts: the construction of laws and the construction of buildings. When the patterns of social order are established, the empathy maintained with tensegrity or presostatic forms comes from understanding the existence of reciprocal forces; forces organized as a group or laws that are necessary to create equilibrium. In these forms, a repetitive pressure could place each member under the same threshold of stress that, in our view, is expressed in the reciprocity of burdens and rights, as in the responsibilities for the balance of the political, ethical and moral system. Highlighting the relationship between collective burden or stress with the idea of culture stifles the freedom of the individual collaborator; this is a submission to group laws without space for any laxity within minimum ratios or tolerable maximums. Without this condition neither statics nor states can exist. In the case of tensegrity structures as static paradigms, they will not remain upright, and in the case of socio-political structures, they will not be appeased.?

For all the above, the conclusion indicates that belonging to a tensegrity unit is to be in touch with the tensions received, coordinately and cooperatively sensitized with the systemic whole and to be capable of reacting to the group tension. A deformation of global balance and/or collective order implies that someone is relaxing and someone is overworking. A state of overwork on the part of some members leads to an increasing rigidity that risks the integrity of the whole with the rupture of the overloaded elements. For the case of statics, when this increase in rigidity is produced, one can also observe the formal expression of such over-effort through the formal appearance of the built whole.

\section{CONFORMATION, META-STABILITY AND} ORGANLESS BODY

The Swiss artist, Paul Klee, had an unusual sensitivity to nature that, from his perception, never stopped moving. Klee said that nature was a great vehicle. This, in constant movement, was exemplified in his paintings and puppets through the rolling waves, the growth of plants, the genesis of a sound or through the stresses of the tight-rope walker as he walks through the clouds (fig. 6). In 1924, in his Elemental creative theory, he introduces the concept of conformation ${ }^{8}$ that has a vital importance for the understanding of his work where he supports his ideas on the instability of form and the perpetual movement of things. The conformation could be explained as the constant process of the evolution of form. This means that the form is not static but is produced taking time and becoming into account. During his time as a Bauhaus master, he emphasized drawing to covey the evolution of form (Klee, 2013). Form ceases to be a mere silhouette or stable outline to be a process of transformation in time and sensitive to all scales.

Finally, Klee explains it very directly:

The shape determines the form and therefore is above it. Thus, the form should never nor in any place (...) be considered as a result, a finish, but a genesis, an evolution. Good is conformation. Bad is the form, the form is final, is death. Conformation is movement, is action. Conformation is life. (Klee, 2013, p. 56).

Moreover, Gilbert Simondon, French philosopher, addressed the stability problem from the fields of physics, chemistry and biology and thereby developed his doctoral thesis Individuation in Light of the Notions of Form and Information published in 1955. The text addresses the theme of individuation, an old problem in art and philosophy defined as that which makes an individual thing completely unique (Simondon, 2009). Thus, Simondon proposed a Pharaonic challenge: attempt to explain how things "become." He began by making a critique of Aristotle's hylomorphic scheme: Simondon found it to have limitations. The accidental collision between matter and form did not convince him. For him, accidents did not exist. He fervently believed in the art and order of energy processes. Therefore, in this Aristotelian duality, where only matter and form would exist during the individuation process, he introduces the notion of energy. So we could say that individuation is formed by the relationship between matter, form and energy.

At this point we can present the concept of metastability. Simondon explains that "(the individuation) has not been thought out and described adequately due to that we know of only a single form of equilibrium, the stable equilibrium; we are unaware of a metastable equilibrium; it was implicitly assumed that the being was in a state of stable equilibrium; however, the stable equilibrium excludes the future [...]" (Simondon, 2009, p. 28). From this position new systems of equilibrium can be conceived, systems that escape from the dualist scheme of movement/ rest and stable/unstable, to open a field of possibilities of evolutionary states of being. This critique of classic stability

introduces a series of notions like the "potential energy of a system, the notion of order and the increase of entropy"

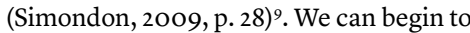
assess the dynamic processes themselves rather than the outcomes.

Metastability allows us to understand that states of form are neither definitive nor isolated. If we look at the stained glass of the gothic cathedrals, we will see that they are wider at the lowest part. Even glass, a symbol of perfect form and balance, is not eternally stable. It flows over time. Glass is metastable and, as such, achieves different states of equilibrium over time. Perhaps what is most interesting in this image, applying it to the problem of representing tensegrity, is the idea of considering a metastable system, loaded with potential, explains itself as a group in which matter, form and energy preexist in the system and evolve over time.

In this system there is a global conception of the group that implies an internal and continuous resonance between all of its elements. The idea of considering them separate results from a practice of the modern method which tends to continually separate the elements that make up the systems, but this individuation principle cries out to rejoin and, as such, for a global and continuous consideration. Because if the qualities of one are codified or informed by the possibilities of the other and vice versa, one must consider the existence of a capacitating energy of movement or state change for it to leave its inert state ${ }^{10}$. In metastability the being is understood as a process and not as a stable form.

Gilbert Simondon ${ }^{11}$ proposes that the metastability idea is found in the simple molding of a block of clay. For him, the formation of a brick is far from the being represented by the Aristotelian duality between matter and form. Simondon presents a beautiful metaphor: the hylomorphic scheme would be one that a man perceives from outside the studio, so he only sees the beginning and end of the process, the step from clay to brick. And in fact, to understand metastability, it is not enough to go into the factory and work with the craftsman but to enter the mold itself (Simondon, 2009). Clay is a material with the ability to be molded, to allow displacement in its composition. However, upon being modeled, it does not suffer arbitrary displacement but its plasticity is ordered according to defined forces that establish the deformation. Now we understand Simondon's critique of Aristotle. There is no accidental collision. There is nothing arbitrary. Everything requires a precise and rigorous order. A precise order that, as with tensegrity, is a basic and fundamental 
plasticity. All matter is a deformable reality, i.e. a reality that is not a defined form but all undefined forms together and dynamically because forces are being deposited in it constantly. But it is not enough that the clay is plastic, it needs a worker to apply force to the mass, force that the clay will distribute to each sheet in relation to the other. Everything changes position, fills the mold, activating potential energy. The mold does not impose the form but stabilizes it. The relationship between the clay particles is almost as strong as the relationship of the clay to the wall.

For complete compression, it must be emphasized that the mold does not impose, as it is not a relationship between inert matter and form that comes from outside, but is a process based on the positive communication, as a particle society, in reciprocal interaction. In a determined moment the limit will appear, i.e. the mold that creates the impossibility of displacement in a determined direction. Let us suppose that the brick is dried by the heat and is formed; the metastability has found one of its possible states of equilibrium. What has really happened is that one of its forces has been annulled. The clay after being molded is the mass in which all the deformation forces are found, in all parts, equal and opposite forces that balance them. Thanks to the metastability of the system, it can be guaranteed that this performance between energy, matter and form has achieved one of the final possible forms where any action had an impact on all the others. That is, the evolution of each molecule impacts the future of all points in all directions. The molding of a block of clay and the manufacture of a brick are expressed in this text as a paradigm of a complex and metastable state of tension.

Through the brick we have seen how Simondon addresses the origin of physical objects, but we will also study the individuation of living beings. For Simondon, the living implies a notion of interiority. The interiority understood as those forces that each living thing carries within; these are forces that are in constant movement, continuously making up our body, at every moment. Something like a constant individuation in time and evolution. Evolution is a transformation, a process of state change that never ends. The notions of interiority and evolution leads us to the brightest student of Simonson, also a French philosopher, Gilles Deleuze. While Simondon makes his point on "metastability" from the physical perspective, Deleuze makes his from the field of art.

Gilles Deleuze taught classes and forms part of the revolution that took place in the Parisian university of Vincennes. The Vincennes experiment center opened its doors in 1968 to reinvent a university education model that was represented only by the old Sorbonne. It was opened in December 7 , 1969 as an intellectual safe haven with the intention of meeting the expectations for the changes that never took place in Paris in May of '68. Vincennes was like a self-managed educational utopia based on the reciprocal efforts of students and teachers. It was a tense system where its existence was reduced to the solidarity, coexistence and democracy of all the parts. There was no entrance exam for admission nor tests, and self-evaluation was encouraged. The professor avoided lectures to be the advisor of a collective work and the students freely chose the subject matter. The instructor's proposals were also discussed here. In short, it was an open system capable of adapting to constant variation. In Vincennes the most innovative and radical thinkers of the time coalesced; Michael Foucauly, Gilles Deleuze, François Lyotard, Jacques Lacan, Michael Serres and Nicos Poulantzas.

Gilles Deleuze was a philosopher enamored with life, leaving static objects aside to focus on bodies. Bodies fascinated him, because they never stopped moving. He critiqued the stable forms as bodies without organs. When Deleuze uses this complex term, he is making a critique not so much of the function of the organs in the body but of the organization of them, to stable forms, to the order and the predictable function that the organs follow in the varied functions of the body. Let us say that this order, this rigid establishment of the organism's way of being, is most certainly detestable for the French philosopher.

Deleuze published an essay on the body without organs in the book A Thousand Plateaus: Capitalism and Schizophrenia. The header image of the text is that of a Dogon egg which he explains as a space of intensities (fig. 8). The body without organs to which Deleuze refers to is an egg. It is a place full of potential without yet a stable form, where everything is tensile forces, energies and multiplicities. The body without organs ignores the substance and the form, basing itself on "the relationships of speed and slowness between unformed elements, and in the compositions of intensive corresponding affections" (Deleuze, 1988, p. 561).

From the field of art, Paul Klee also discusses the egg as a place of potential: "What is the egg. It is clearly a square. The square is an egg, the matrix of the dimensions" (Deleuze, 2007, p. 40). The matrix is understood as a place where the dimensions are born, i.e. where that which can be measured but as yet there is nothing. As a metastable body, the egg is where all these internal tensions that make up the individuation process are found. From this idea, it is perhaps easier to understand Paul Klee's maxim on art: "It is not to reproduce the visible, it is about making it visible". And what is the invisible for Klee? The invisible are forces and energies, and for Klee this is all very obvious. Let's look what he says in his book The Theory of Modern Art: "(Where do you get so much energy? Vain question from a simpleton). 'Peace on earth' is an accidental pause in the movement of matter. To have this fixation of a first reality is pure illusion, [...]" (Klee, 1976, p. 59). Deleuze finds these invisible forces in the work of Michelangelo and he writes: "Now, what does it mean to paint the broad back of a man? It is not painting a back, but painting the forces exerted on a back or the forces the back exerts. It is painting forces, not forms" (Deleuze, 2007, p. 71). These words also allude to the Irish painter, Francis Bacon (1909-1992), whose work was one of Deleuze's main weaknesses. Bacon paints forces and bodies, bodies that are sensitive (hypersensitive) to the forces around them. Bacon's paintings are not mere stable figures, but bodies ${ }^{13}$, understanding the body as a place where internal and external forces are applied, the matrix, the egg. Body situated in evolution and formed by it.

Again, the story argues how the stable form is threatened in favor of a living capacity of the form, away from molds or limits. The form is not drawn (built). The tensions (forces and energies) affect the body. Bacon is passionate about sleeping bodies; where no forces appear to exist, as if they could be seen, Bacon paints them.

LISTENING TREE OR ODE TO FAILURE The ideas unfolded along the previous points have established relationships between philosophical thought, aesthetics, statics, state and energy, as well as enthusiastically praise the empathy of its possibilities for formal relationship. The same concept and descriptive details have cleared up the existing relationships between the various spheres of knowledge, and some have been used to explain the others. Finally, in this article, tensegrity is used as an idea that is expressed efficiently in both the technique of erecting structures to the constitution of the State. Both coincide with the idea of potential energy that Gilbery Simondon developed to explain the scope of metastability as an intermediate point between the stable and the unstable. The idea of metastability aids in thinking of tensegrity with more tools, better concepts and greater thematic diversity because the metastable balance is a transitory state of stabilities.

On the contrary, to think of tensegrity from the paradigm of stability and not from metastability, reduces it into a field that fails to incorporate sensitivity to the transitory state in which this load composition, tensions and the internal activity of the system are assembled. Perhaps this situation is not visible, because at the end of the day, when speaking in the context of statics and State, it means evading all the evolutionary concepts; for statics, for abusively desiring a permanent and definitive state, and for the State, for its desire to preserve equality over historical evolution. But in both cases, and above

all the case of the State, the contingency, mobility and the fluctuations impede the stable balance, while in statics, it is converted into the desire to create the image of stability the State does not offer. Mainly, through its tectonic appearance, architecture solves the paradox of offering an excessive stability for a world that is essential mutable and truly fragile.

Now, it is desirable to achieve the commitment of carrying out a structural model of tensegrity that highlights the need for reciprocity of forces for the existence of a transitory, metastable stability, operating in the open design of the system relationships (fig. 9).

Regarding this proposal, it would be worth observing as the partial failure of reciprocities would make the total balance vulnerable, that is, the value of the existence of an order and its capacity for evolution. If a tensegrity system would take seriously the faithful representation of statics updated to the reality of the world, it would have to strive to sharpen its constructive solution to the limit in which any loss of belonging to the systemic order would cause the complete collapse of the tensegrity unit. This would mean achieving a state of tensegrity purity that would imply working exclusively with elements for the force diagram: simple traction forces, simple compression and the discontinuity of compression forces. All this would establish a permissive order for bring together the image of statics to the image of the state, both being fragile, changing and complex.

In 1953 French architect and engineer Robert Le Ricolais received the commission to design a long-range, high precision cannon for the French army. Increasing their length, which increases their weight and provokes a deviation from the sightline, makes the long-range cannons, passing from straight to slightly curved, thus losing precision. The precision problem is resolved by seeking out increased length while at the same time achieving rigidity and lightness. Le Ricolais offered a solution that consisted of compressing a mast with a set of peripheral cables. The tensed cable joined the two extremes of the bar, and along the guideline he placed a set of rods perpendicular to the mast to offer a collection of forces to the axis that reduced the amount of buckling while they were compressed between the cables and the mast. If the buckling length decreased without problems, the sight line could be perfectly straight and would tremendously lighten the group. 
opened upwards, they were surrounded by circular rings that built an inverse cone that connected to the masts by means of radial rods perpendicular to the central axis of the tower. This was not a tensegrity model; it was more of a system compressed by its own weight and the tension forces of a family of peripheral cables.

In tensegrity spheres, the failure of a single element implicates a partial failure of the form since the sphere can respond in various directions as well as the inherent capacity of its geometry to respond structurally as a collection of rings, a dome or an arch. For these characteristics, it would never completely fade. On the contrary, a tensegrity tower model could be the most appropriate for stimulating the formal empathy between diverse concepts. The tower idea, apart from recovering atavist images on the act of erecting, symbolizing and elevating, manifests a collapse along the vertical axis by fading away, a descent of the entire assembly toward a fulcrum due to the effect of gravity and therefore an emphatic image of failure given that being straight is what makes a tower what it is.

Returning to Le Ricolais's previously described proposal, one would only have to transform it functionally toward a tensegrity model. If we remove the connection between the rings and poles, the rings are literally suspended in air while working unitarily with an internal rigidity proportional to the rigidity of the whole. Similarly, if we transform the rings into polygons (fig. 10), the structure presents as an assembly of rods and cable, more effectively responding to the rectilinear path of the loads. The nodes are articulated so that the plane of the polygonal rings only works in compression (fig. 11). Then, the cables are organized in four opposite directions, two by two, passing through the geometric center of a metallic disk and the whole result is equal to the sum of the compression on the rods converging toward the fulcrum of the assembly. Resolved like this, the whole structure will be predisposed to failure approaching maximum stability between two extreme opposites: to stay standing or to fall completely to the ground.

Following this reorganization and having trivialized stability principles used to build long range, high precision cannons, would have passed from a stable to radical metastable state. A state where failure was certain for a milimetrical deformation from the initial solution, because we can see that the nodes are not embedded and the rods remain in compression by a ring that does not fit between ends of the rods by a difference of millimeters; this difference is proportional to the possible bowing and twisting of the wood rods when they change hygroscopic state, as illustrated in the Cracking Box by David Nash.

In the year 1992, David Nash allowed the wood's nature to finish his sculptures. Cracking Box, a perfectly square cube of wood, was left to its formal evolution every time the wood reacted to a humid environment. After some months, the box expressed the formal discord of the cube through fissures, ripples and breaks that made the box a double geometric shape: the origin and the arrival. This idea translated to Le Ricolais' banal tower proposal, eventually leads us to complete the concepts, forms and principles for the tensegrity structure that we announced as the proposal: a structure that we call Listening Tree (fig. 12).

Listening Tree is a tensegrity structure that is built with wood and metal. The wood used for the rods is cut against the grain, finer to the outside than the center and with a curved section lengthwise that responds better to compression (fig. 11). Once the group is assembled, it is given over to its evolution, knowing that wood is loaded with interna potential energy and an active capacity for transformation in relation to states of relative humidity. In turn, these rods are coordinated with the whole of the structure through the collective stress that facilitates the tensile forces. Internal tress, load and tension permit the equilibrium of the system. When the Listening Tree is introduced in the Sierra de Guadarrama environment, at the feet of the Cerro de San Pedro in Madrid and outside the workshop where is has been prepared, a process of internal deformation to adapt the wood to the environment will slowly create a diverse form in each rod (fig. 13). As it is reaching equilibrium, Listening Tree will continue to warp in new and diverse ways until reaching the limit of its existence. That form in which passes over the threshold of its last possible equilibrium and bubble up and vanish in to the air.

\section{CONCLUSION}

The proposed in this article is open. It does not demand a certain reading, rather is positioned in an intermediate point between all concepts and themes unfolded to speak of the same thing: the potential to form processes and, consequently, the manipulation of thought in discussing what the title calls the abuse of the stable forms as an assumed practice.

In the case of the Listening Tree it would be insignificant to understand its failure as a form of expressing fragility. A kind of fragility inherent in the structure of a system, that makes purity visible as a strange state. A radical purity without which equilibrium cannot be maintained and that shows the potential state of its stability as a transitory form of becoming. That is, it is offered as a metastable equilibrium between two possible points: maintaining equilibrium associated with the atmospheric conditions or fail by giving into gravity. Thus, it is seen as something hypersensitive to the changes of the environment it inhabits. Finally, Listening Tree vanished into the air on November 16, 2011. ARQ

Notes

1 In the world of laws, recipes and customs are respected in the oldest societies. They are untouchable in the most archaic societies. 2 The chirotop or the human hand domain, the phonotop or that of the vocal sound, the uterotop or the maternal realm, the thermotop or the comfort experiences, the erototop or the place of erotic energy transferences, the ergotop, the sphere of parental or religious authority; the alethotop or learning group within the truth realm, the thanatotop or the ancestors domain, and the nomotop, which represents the social agreements on the politics sphere.

3 From the expression Tensión Integrity Structures by Buckminster Fuller.

4 Aside from the debate on the author of the first tensegrity structure, it must be said that Ioganson accomplished two things, simultaneously and without precedent: a revolutionary static principle and a revolutionary socio-political state. 5 An alternative planted a displacement of the centers of gravity and formal structures without surpassing the problem of gravity as a hegemonic theme and sought a visual imbalance or energy in the form. Definitively, a a first critique of statics. 6 The Russian Avant Garde was involved in this technical and productive transformation, reaching their peak between 1923 and 1926. In the 1925 International Exhibition of Modern Decorative Arts and Industries in Paris the Russian show won severa medals.

7 That the collective stress be the common pattern for stable functioning, the reaction to the unexpected or adaptation to the mobile, allows for thinking in how or under what conditions the constant increase of internal tensions are made which must be overcome without the tiring experience of continuous tension. The institution and routines are modes of social operation for the sublimity of these continous states of tension. We can assume that these two modes for regulating continuous tensions sacrifice freedom of choice and what disappears is the overwhelming freedom of choice for the individual that expects the state of balance. And so, this individual flees from the demanding task of the originality of his actions and the misery of the lack of ways. Unburdened from the continuous pressure, energy is freed for other tasks. To the architects, each time they undertake a formal idea, they must defend each step in a web of productive relationships focused toward the same objective. The division of work posed by modernity obliges us to the structuring of ordered internal tensions in a self-stable pattern in which all components are knowingly aware of the dependence of such agencies for collective achievement. The complementarity between the parties creates a tension demand unifying the labor division between coalesced agents. This load modulation of increases, decreases and tension having been effective, is made essential for all successes, given that such achievements are the result of creating tensions between sensibilities of diverse disciplines and coupled to a beauty created through the disciplinary rigors of many affiliated individuals under the same tension at each step.

8 Klee's concept of configuration must be related to Jacques Monod's ideas on teleonomy and Henri Bergson's élan vital. In Monod, the concept of teleonomy appears to explain the formation that accompanies an autonomous morphogenesis that as a project carrier loaded with information allows fo things to be, administrating the energy contained within them (Monod, 1981). In Bergson's words, the matter tends to fall and life tends to rise, driven by a élan vital that never ceases and in whose collision produces the being (Bergson, 1977). These last words, while still being intuitive and building a clear image of the diverse states of energy in evolution, help one to see what can be undestood between life and death translated to states of pure energy. 9 In the first proposal, he will not only speak of the existing physical individuation bet also the vital and psychic individuations as in the case of resolving a metastable system. In this proposal, the formula illustrates the states of a system such as over-fusion or over-saturation that preside in the genesis of the crystals. Considering them as clear to give solvency to the metastability concept is not complex enough to encompass the problem of individuation in an exhaustive way. The metastability is a kind of weak stability that progresses toward strong states of stability each time a significan evolution in produced in the being. This implies an evolving and continuous state, that is, the presence of becoming. 10 Therein lies our critique of stability, in the impossibility of ontogenesis, that is, in the impossibility of a being built though a process. In the physical individuation, matter passes into a kind of active process of constant becoming and resulting in haecceity object that has relational and insubstantial properties.

11 Gilbert Simondon in his book The individuation to the light of the notions of form and information will explain within the first paragraph of the first chapter the physical individuation applied in the forming of the brick, rejecting hylomophic duality incorporating the notions of energy and becoming to understand this continous process (Simondon, 2009, p. 48-56).

12 According to basic chemistry principles, a repetitive structures are considered crystals. 13 "When they say that everything is body, they mean to say very simple things. For example, a circle does not extend in the space in the same way if it is wood or if it is marble. "Everything is body" will mean that a red circle and a blue circle extend into space differently. This deals with the tension. A thing that painters know very well. When they say that all things are bodies, it means that all things are defined by tones (en Greek, tone means tension) the contracted force that defines something. If you cannot find a kind of contraction, the embryonic force within something (the body), you do not know it" (Deleuze 2004, p. 381)
RAFAEL BENEYTEZ DURÁN | Architect, Escuela Superior de Arquitectura de Madrid de la Universidad Politécnica de Madrid, 1998 and PhD Internacional Candidate, 2009. Between 1999 y 2007 collaborated at Rafael Moneo Studio being Project and Works manager. He was design studio professor and director of Aesthetics and Composition at the Escuela Superior de Arquitectura y Tecnología de Madrid; he has been visiting faculty member at the universities of Würzburg, Limerick, Delft, Harvard, Barcelona and Münster. Currently he is associated professor at the Universidad Politécnica de Madrid, develops his doctoral thesis on atmospheric issues in architecture and runs his practice Z4Z4 AAA (Architecs Activities Associates).

Víctor MANUEL CANO CIBORRO | Architect, 2012 and Máster en Proyectos Arquitectónicos Avanzados, 2013, Escuela Técnica Superior de Arquitectura de Madrid de la Universidad Politécnica de Madrid. He is connected to the Grupo de Investigación 